\title{
Comparing Human Robot Interaction Scenarios Using Live and Video Based Methods: Towards a Novel Methodological Approach
}

\author{
Sarah Woods, Michael Walters, Kheng Lee Koay, Kerstin Dautenhahn \\ Adaptive Systems Research Group, School of Computer Science \\ University of Hertfordshire \\ College Lane \\ Hatfield, UK, AL10 9AB \\ \{S. N. Woods, M.L.Walters, K.L.Koay, K.Dautenhahn\} @ herts.ac.uk
}

\begin{abstract}
This paper presents results of a pilot study that investigated whether people's perceptions from live and video HRI trials were comparable. Subjects participated in a live HRI trial and videotaped HRI trials in which the scenario for both trials was identical, and involved a robot fetching an object using different approach directions. Results of the trials indicated moderate to high levels of agreement for subjects' preferences, and opinions for both the live and video based HRI trials. This methodology is in its infancy and should not be seen as a replacement for live trials. However, our results indicate that for certain HRI scenarios videotaped trials do have potential as a technique for prototyping, testing, developing HRI scenarios, and testing methodologies for use in definitive live trials.
\end{abstract}

\section{INTRODUCTION}

In the course of our work for the COGNIRON Project [2005], we are primarily interested in the research area of Human-Robot Interaction (HRI), in particular with regard to socially interactive robots. An excellent overview of socially interactive robots is provided in Fong et al.[1]. We are primarily interested in the human perspective of how robots could be useful in domestic environments; in particular the roles, tasks, and social behaviour that will be necessary for robots to exhibit in order to integrate into normal domestic situations. In order to study human-robot relationships, we typically run HRI trials using carefully devised test scenarios, where human responses and opinions can be collected using a variety of methods. HRI trials are particularly difficult to develop if they should involve complex robot behaviours that nevertheless need to be reliable and replicable for statistical comparisons, various robot platforms etc, in addition to large sample sizes of subjects, balanced for age, gender, cultural background etc. In order to address this situation, we are interested in verifying whether videotaped HRI trials for various scenarios could be used in certain situations instead of live HRI trials, or as a complementary methodology for live trials.

\section{A. Human Robot Interaction Trials}

To date, we have conducted various live HRI trials with human scaled PeopleBot ${ }^{\mathrm{TM}}$ robots [2][3][4] Other researchers have also conducted similar HRI trials with human sized robots including Dario et al. [1], SeverinsonEklundh et al.[5], Kanda et al.[6] and Hinds et al.[9].
However, most HRI trials to date typically are characterised by relatively small sample sizes [6][7][8]. Our largest HRI study to date involved 28 subjects and took about 2 months to carry out. We have run several larger HRI trials, but this has been at the expense of compromising the trial conditions (audience noise, lack of extensive post trial questions etc). Running a live HRI trial under controlled conditions therefore requires a major commitment of time, resources and personnel to ensure that statistically valid results are obtained. HRI studies in general are at a stage where there is not a large body of prior work to guide the design of large scale live trials. This means that most studies are highly exploratory. Many initial assumptions are based on those expected from human-human interactions, which we and others have found do not always hold true for human-robot interactions [9][10][11][12]. It is sometimes difficult to justify speculative or exploratory trials where, by their nature, there is a higher probability of the predicted assumptions not being met, being inconclusive or irrelevant. Before committing to a major trial it is essential to run pilot studies to test the proposed methodology. It would be advantageous to have a methodology in place where trial predictions could be piloted and tested, before developing and executing full live trials.

\section{B. 1.2 Video Based HRI Trials}

To overcome some of the drawbacks of live HRI trials, the feasibility of running HRI trials using video footage rather than a full live interaction was considered. Although this methodology would certainly be inferior to a live HRI session, it was hoped that it would yield valuable results towards the development of live trials. Kidd [13] found no significant differences between subjects' ratings of personality traits for 'present' and 'remote' (through video) cases of an interaction with a robot head. Shinozawa et al. [14] reported that comparing a robot's recommendation behaviour with an on-screen agent's, for human decision making, depended on the interaction environment and that geometrical consistency between the interaction environment, and robots and on-screen agents was important. Paiva et al. [15] reported that synthetic (cartoon-like) characters in virtual environments were readily empathized with by children as they enacted various scenarios. This provides supporting evidence that believable relationships can be created through the medium of video. Using videos of robots, which are more realistic than virtual or synthetic 
characters, could result in HRI trials that are even closer to resembling real live interactions. Video based HRI trials have the potential advantages to: 1) reach larger numbers of subjects as they are quicker to administer, 2) easily incorporate subjects' ideas and views into later video trials simply by recording extra or replacement scenes into the video based scenarios, 3) carry out trials exposing groups of subjects to the HRI scenario simultaneously, 4) prototype proposed live trial scenarios to avoid wasted effort and test initial assumptions, 5) allow greater control for standardised methodologies (i.e. exactly the same robot behaviours, exact trial instructions etc.). As this is an unexplored area of HRI studies, it is first necessary to confirm whether video based HRI trials are able to provide comparable results to live trials, and also under what circumstances. A pilot study using both live and video HRI trials was developed to begin exploring the following main research questions:

1) Will video based HRI scenarios provide results that are comparable to results obtained from live HRI trials?

2) Under what circumstances would video based trials provide comparable results to live HRI trials?

3) What are the likely limitations of video based trials in gaining valid human responses to HRI scenarios?

\section{Experimental Method}

The Video-Live Trial (VLT) pilot study was carried out in a converted conference room. The chosen scenario involved a robot using different approach directions to bring a seated subject an object. The aims of the trial were to find out about subject preferences for the robot approach directions. The room was partitioned into two areas; a video trial area and a live trial area. There was a gap in the partition, so that it was possible to move between the two areas (see Fig.1) but not possible for subjects to see the other area while carrying out the respective video or live trials. The live trial area resembled a simulated living room with a chair and two tables. The subject was seated in the chair throughout the live trial which was positioned halfway along the rear wall (point (9), Fig.1). To the left front and right front of the chair, two tables were arranged (with room for the robot to pass by) in front of the chair. One of the tables had a television placed upon it; the other had a radio and CD player.

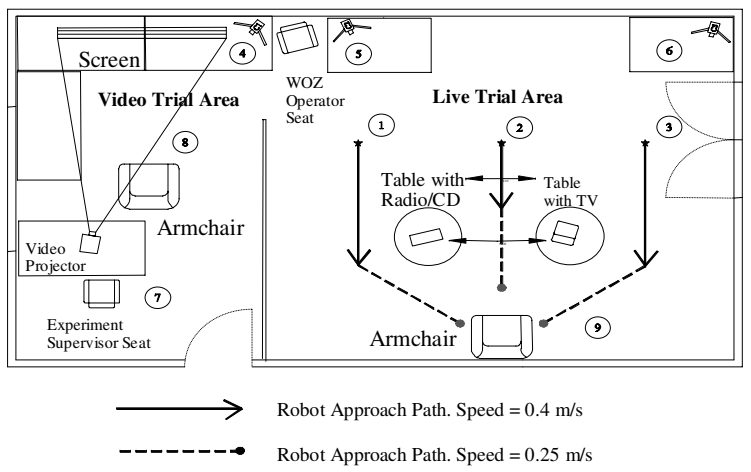

Figure 1. Diagram of video and live trial experiment areas

The robot was driven to the appropriate start position by an operator seated at a table in the far corner of the room.
Subjects were told that the robot would be controlled by the operator while it was driven to the three start positions, but would be approaching them autonomously to bring them the TV remote control. This was reinforced as the operator made notes and did not press any of the robot control keys (on the robot control laptop) while it approached the subject.

The video trial area contained a video projection screen and projector for playing the video HRI trial scenarios. The videos were all recorded in the live trial area, with an actor playing the part of the subject. The actor was male, and the narration voice which introduced and set the scene for the HRI trial scenario was also male. The videos were recorded using a mixture of first and third person points of view. The third person views showed the overall positions and actions of both robot and (actor) subject. Then by switching to a first person view (from the perspective of the subject sitting in the chair as the robot approached) a viewer saw the robot approaching in a way that was as realistic as possible and could gain some spatial perspective (see Fig. 2 for example screen shots).

\section{The HRI Trial Scenario}

An identical scenario was used for both the video and live HRI trials and took place in a (simulated) living room (Fig. 1). It was introduced either by the experiment supervisor for the live trial, or by the narrator for the video based trial. The context was that the subject had arrived home from work and rested in an armchair (point (9), Fig.1). The subject then asked the robot to fetch the remote control. It was explained to the subject that the robot was new to the household and it was necessary to find out which approach direction the subject preferred; either from the front (2), the left (1) or the right (3) (see Fig. 1). In order to justify the robot fetching the remote control, one of the tables had a (switched off) TV set upon it. The other table had a CD-Radio unit. Our expectations prior to the trials were that subjects would prefer the approach from the front, since the robot was then fully visible at all times.
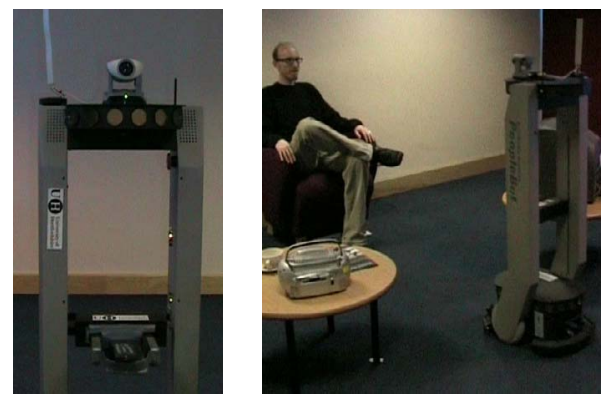

Figure 2. Examples of first and third person views

\section{E. Experimental Conditions}

We were aware from a previous demonstration that the TV was a natural focus of subjects' attention and could have influenced the choice of preferred robot approach direction. Therefore, half the trials (for both live and video versions) were carried out with the TV on the left hand table, and the other half with the TV on the right hand table.

Each subject experienced the robot approaching from three directions: front, left and right in a counterbalanced 
order sequence covering all six possible permutations of the three robot approach directions. This was used for both video and live trials. As a consistency check, the three robot approach directions were also repeated (in a different order) for each trial. In order to counterbalance for effects due to the order in which subjects experienced the video and live trials, we exposed half the subjects to the live trial first, then vice versa for the other half of the trials. Fifteen subjects ( 9 (60\%) males; 6 (40\%) females) individually participated in the study. The mean age of the sample was 33 years (range 21-56 yrs). Only one subject was left handed. Four subjects were secretarial staff from the University of Hertfordshire, 5 subjects were MSc students studying 'Artificial Intelligence', and the remaining 6 were research staff in the Computer Science Department at the University.

\section{F. 2.5 Procedure}

A short introductory questionnaire was used to gain the necessary demographic and personal details from the subjects. At the end of each video or live HRI trial a short questionnaire was used to assess the subjects' views on approach direction, approach speed, stopping distances, comfort levels and practicality for the different approach directions. After both video and live trials had been completed, subjects participated in a semi-structured interview with a psychologist. The interview was carefully designed so that no leading questions were asked. The interviewer was able to follow up answers to gain a deeper insight when necessary. The main purpose of the structured interview was to assess the subjects' views on the trial procedures and methodology, establish any weaknesses and find out how the trial could be improved from the participants' point of view. The subjects' reactions to both live and video based HRI trials were recorded on video tape.

\section{RESULTS}

\section{A. Approach Direction most preferred and least preferred.}

Results of the trials clearly demonstrated that the least preferred approach direction was the front approach, for both the live and video trials. The right approach direction was the most preferred for both the live and video trials, and the left approach direction was preferred equivalently for both live and video trials. Only one person could not state a preference for any of the approach directions based on the video data. Fig. 3 illustrates that there was approximately $58 \%$ agreement for the different approach direction preferences, between the live and video trials. $36 \%(\mathrm{~N}: 5)$ of subjects stated that they preferred the right approach direction in both trials, and $22 \%(\mathrm{~N}: 3)$ rated that they preferred the left approach in both trials. Surprisingly, no subjects preferred the front approach direction in both trials. Where agreement was not found between the live and video trials, this was predominantly found for changes in preferences for the left and right approach direction. One subject $(7 \%)$ stated that they preferred the front approach for the live trial, but the left approach direction for the video trial. One subject (7\%) preferred the left approach direction for the live trial, but the front approach direction for the video trial. Two subjects (14\%) preferred the left approach direction in the live trial, but the right approach direction in the video trials. Finally, 2 subjects (14\%) preferred the right approach direction for the live trial, but the left approach direction for the video trial.

Cross-tabulation tables were produced to calculate the percentage of agreement between subjects' approach direction preferences for the live versus video trials. McNemar-Bowker test, a nonparametric test was carried out to detect changes in responses between the live and video trials. No significant effect was found indicating that there were no significant differences in subjects' approach direction preferences in the live and video methods used.

Fig. 4 demonstrates that there was $85 \%$ overall agreement between subjects' ratings of the approach direction they least preferred for the live and video trials. $77 \%$ of subjects stated that they least preferred the robot front approach direction in both the live and video trials. One subject (7.7\%) least preferred the left approach direction in both trials. No subject rated the right approach direction as least preferred in either the live or video trial.

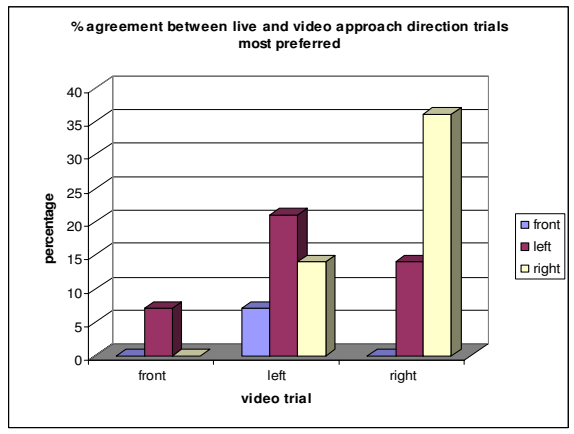

Figure 3. Percentage agreement between approach direction most preferred for the live \& video trials

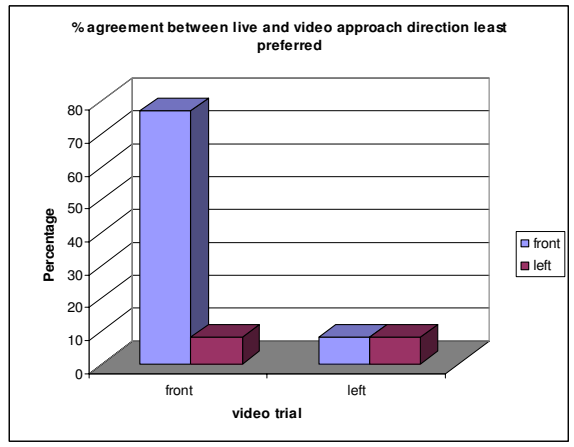

Figure 4. Percentage agreement between approach direction least preferred for the live \& video trials

Only one subject $(7.7 \%)$ who least preferred the front approach in the live trial, had rated the left approach direction as least preferred in the video trials. One subject $(7.7 \%)$ least preferred the left approach direction in the live trial, but the front approach direction in the video trial. A McNemar test revealed no significant differences in the approach direction least preferred by subjects in the live and video method robot trials.

\section{B. Robot Stopping Distances}

1) Front Approach Stopping Distance; 
The robot was set up to stop automatically at a distance of approximately $0.50 \mathrm{~m}$ from the leading edge of the robot and the nearest part of the person's body. There was some overshoot or undershoot giving a tolerance of $+/-0.15 \mathrm{~m}$. Overall, there was 66\% agreement between subjects for ratings of whether the robot's stopping distance was too close, about right, or too far for the live and video trials (Fig. 5).

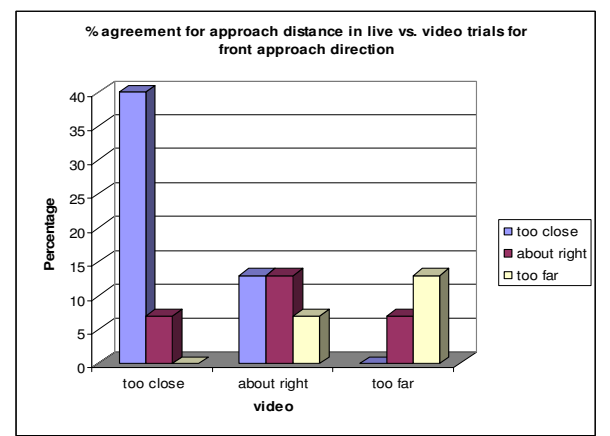

Figure 5. Percentage agreement for robot stopping distance from subject for front approach direction

Figure 5 indicates that a majority of subjects rated the front approach stopping distance as too close. For subjects who rated the front stopping distance as being too far, we observed that these subjects usually had their legs stretched out in front of them. This caused the robot to stop when it reached the subject's feet, rather than moving close enough for them to reach the TV remote control (due to the robot's stopping safety mechanism). Overall, there was $60 \%$ agreement between the live and video trials for subjects' ratings of the robot's stopping distance for the left approach direction. There was overall $80 \%$ agreement for the robot's stopping distance for the right approach direction in both the live and video trials.

\section{Robot's Speed during the Live and Video Trials}

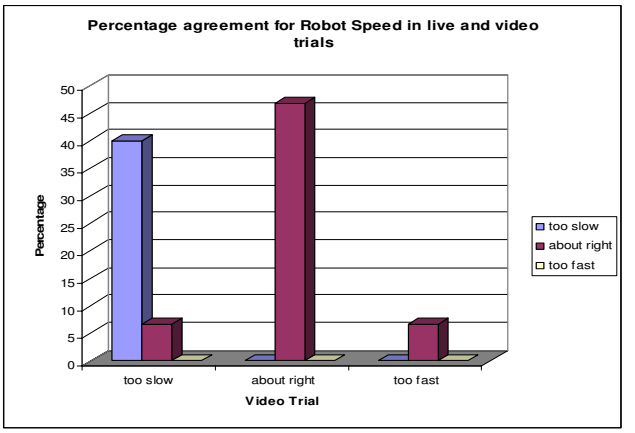

Figure 6. Percentage agreement for robot speed in live and video HRI trials.

The robot used two speeds; the normal approach speed was $0.4 \mathrm{~m} / \mathrm{sec}$, and the robot slowed to $0.25 \mathrm{~m} / \mathrm{sec}$ when it was within $1 \mathrm{~m}$ of the front of the subject. When subjects were asked to rate the speed of the robot's approach direction, there was high overall percentage agreement between the live and video trials $(87 \%) .46 \%(\mathrm{~N}: 7)$ of participants rated the robot's speed as about right for both the video and live trials, and $40 \%(\mathrm{~N}: 6)$ of subjects rated that the robot's speed was too slow in both the live and video trials. Where agreement was not found, one subject $(6.7 \%)$ rated the robot speed during the live trial as about right, but too slow for the video trial, and one subject $(6.7 \%)$ rated the robot speed in the live trial as about right, but too fast in the video trial. No subjects rated the robot speed as too fast during the live trials (Fig. 6).

\section{Practicality and Comfort for the different Robot Approach Directions}

In addition to subjects rating which approach direction they preferred for the live and video robot trials, ratings were given for how 'practical' they thought each approach direction was for the given task of delivering a TV remote control, according to a 5-point Likert scale $(1=$ not practical at all to $5=$ very practical) (Fig. 7). Paired samples t-tests did not reveal any significant differences between subjects' ratings of practicality for any of the approach directions between the live and video trials [left approach direction $\mathrm{t}=$ 1.47 (14), $\mathrm{p}=.16$, right approach direction $\mathrm{t}=.521(14), \mathrm{p}=$ .61 , front approach direction $\mathrm{t}=-1.08(14), \mathrm{p}=.30]$. This indicates that there were no large discrepancies in subjects' judgments and subsequent ratings of task practicality between the live and video trials. Ratings of the practicability of the approach directions for both the live and video trials were relatively high. The front approach direction received the lowest ratings of practicality for both the live and video trials. The left approach direction in the live trial received the highest ratings of practicality followed by the right approach for both the live and video trials

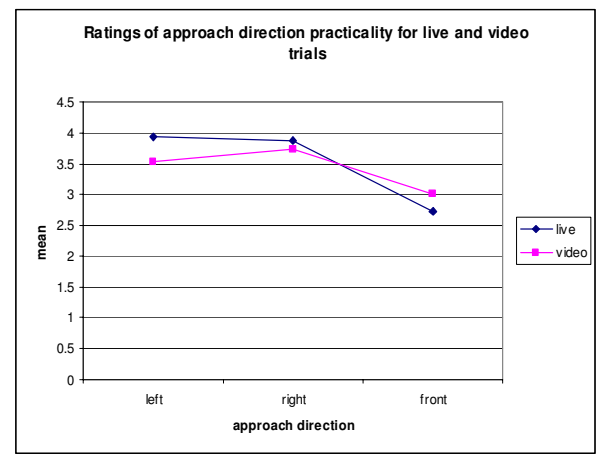

Figure 7. Ratings of approach direction practicality for live and video HRI trials

Subjects were asked to rate how comfortable they felt with the different approach directions for both the live and video trials according to a 5-point Likert scale $(1=$ very uncomfortable, $5=$ very comfortable). Paired samples ttests revealed no significant differences between subjects' comfort level ratings according to the different approach directions between the live and video trials [left approach direction $\mathrm{t}=1.23(14), \mathrm{p}=.24$, right approach direction $\mathrm{t}=$ 1.58 (14), $\mathrm{p}=.14$, front approach direction $\mathrm{t}=-.52(14) \mathrm{p}=$ .61]. This highlights that subjects' comfort ratings were relatively equivalent for both the live and video trials. The lowest mean comfort levels were found for the front robot approach direction for both the live and video trial. The highest comfort level rating was found for the right approach 
direction in the live trial followed by left approach direction in the live trial. It seems that participating in the live trial led to slightly higher (although non-significant) ratings of comfort compared to the video trials. No significant differences were found between the most preferred approach direction and least preferred approach direction for gender, subject handedness (whether subject was left or right handed), and occupation.

\section{E. Realism of the Live and Video Robot Trials}

Subjects were asked to rate the overall realism of the live and video robot approach direction trials ranging from $1=$ not realistic to $5=$ very realistic. The mean rating was 3.47 (minimum $=2$, maximum $=4)$. Subjects were subsequently asked how they would have improved the trials. The comments that subjects made were classified according to those that referred to the set-up of the trial and room, and those that referred to the robot's characteristics:

Subjects' suggestions about how the trials could be improved included a more chaotic and messy environment, more objects and obstacles (as the room was too sterile and clinical), more furniture, and a more busy environment. They would also like the robot to indicate that it understands the person (e.g. by eye gaze), be smaller (as less threatening) and also fetch the remote control itself at start of task (the robot had it in the basket already).

93\% of subjects stated that they preferred the live robot trials compared to the video trials which was not surprising. However, $80 \%$ of the sample stated that they felt the video robot trials were representative of the live robot trials.

\section{DISCUSSION}

The main findings from this study were:

1) The level of agreement between subject responses for the preferred robot approach direction was relatively high $(60 \%)$ between the live and video trials. Discrepancies were mainly due to the fact that subjects did not have strong preferences for either the left or right robot approach direction and sometimes changed these preferences between the video and live trials.

2) Very high levels of correspondence $(85 \%)$ were found for subjects least preferring the front robot approach direction in both the live and video trials.

3) Moderate to high levels $(60-80 \%)$ of agreement were found for perceptions of the robot's stopping distance from the subject, for each approach direction in the live and video trials.

4) High agreement (87\%) was found for subject ratings of the robot's speed between the live and video trials.

5) No significant differences were revealed between subject ratings of how practical and comfortable the different robot approach directions were for both the live and video trials.

6) Subject ratings for the realism of the video trials in comparison to the live trials were moderately high, although 93\% stated that they preferred interacting in the live trials.

These results support findings from an informal earlier study, and also provide additional support for using video methods. We had thought that subjects might find it difficult to perceive the robot distances, and speed through the video medium, but this may not be the case. The non-significant findings for subject ratings of the practicality for the robot approach direction task, and comfort levels between the live and video trials was positive. This is indicative of subjects being able to report on the subjective experience of how comfortable they would feel with different robot approach directions through video footage.

Most subjects preferred the live robot-interaction trials. This was not surprising as live trials seem more interactive ${ }^{1}$, likely to be more fun, and more engaging, compared to watching the interactions involving a stranger on a screen. The embodiment experience of being part of a live-set up is also likely to be much more beneficial for assisting in the perception of speed, distances and different robot movements compared to video footage. However, most also reported that the video robot trials were representative of the live trials. Subjects' overall ratings of the 'realism' of the approach direction robot trials was moderately high and most of the improvements that subjects cited were related to the environmental set-up, and context, rather than characteristics of the robot. According to our subjects' suggestions, our future robot trials should take place in a more naturalistic 'messy' living room set up, which is more representative of a realistic home environment.

The results of this pilot study are in line with the findings reported by Kidd [13]. We are not aware of any further studies to date that have considered the suitability of using video footage for human-robot interaction studies. Naturally, there are numerous limitations of using video footage for HRI studies, and we are by no means suggesting that they should be a replacement for live HRI studies. It could be that the more interaction between a robot and a subject in a trial, the less suitable video trials will be. The timing and synchronization of movements play an important part in regulating and sustaining meaningful human-human interactions. Developmental psychologists (e.g. [16][17]) have shown that while babies happily interact with their mothers via live video, they get highly distressed when watching pre-recorded or replayed videos of their mothers (as it lacks the contingency between mother's and baby's behaviour). However, for the particular research questions that we consider in the context of robot motion planning and approach directions, contingency of robot and human movements plays a less crucial role and thus lend themselves to investigations of video trials.

Only 15 subjects participated, many with a robotics or computer science related background that may have biased the results. However, such subjects are most likely to be future customers of a robot assistant in the home. Nevertheless, naïve subjects who have no prior experience with robots might form an interesting control group in future studies. We limited the sample size for this initial pilot study but given the current positive findings, we aim to replicate the current study with a larger sample size. In future trials, we also intend to incorporate more naturalistic set-ups. The quality of the robot trials could be enhanced if professional camera techniques are adopted and guidelines developed to create video material for HRI video trials. It was beneficial

\footnotetext{
${ }^{1}$ Even in scenarios like ours where the robot's behaviour does not depend on the subject's responses the live situation affords interaction.
} 
that both first person and third person views were used for the video trials as we think that this enabled subjects to get a more realistic perspective of space and distance for the robot.

The current findings offer scope for future work into the feasibility of using video based HRI trials to aid the design and implementation of live interaction studies. We have only considered one human-robot interaction scenario in the current study. It is important to determine whether these results can be replicated and in addition to consider different scenarios. In some ways, the current trial was challenging, as the issues of speed, space and distance were considered. It may be the case that video footage is more comparable to live trials for exploring subject responses to robot gestures, robot appearance, and robot dialogue.

In addition to replicating the current study, a range of different set-ups could be considered. In the meantime, we carried out studies into subjects' opinions towards robot approach directions when the subject is standing rather than sitting, and whether they are sitting behind a desk or not when the robot approaches them with an object. We also used video footage in HRI studies that investigated opinions toward different robotic appearances (e.g. mechanistic vs. humanoid appearance). As the research area of socially interactive robots is relatively new, there are few design paradigms to provide input to this project and others [18]. However, until more studies are carried out using different scenario set-ups and robot behaviours with larger sample sizes, we cannot yet positively conclude that video based HRI trials are a reliable and informative means of gaining data to assist in the future design of robot companions.

\section{CONCLUSIONS}

To conclude, encouraging results were obtained comparing the agreement between subject responses towards robot approach directions for live and video human-robot interactions. This has positive implications for researchers designing future HRI trials, as video trials could be used as a complementary research tool to yield valuable results regarding peoples' opinions towards various aspects of a robot's behaviour and/or physical capabilities. Video trials are more economical compared to live interactions, and allow the designers/researchers greater levels of control and standardisation over the set-up of the trials, which is sometimes difficult when conducting live HRI trials. We hope that our results, while still at a preliminary stage, will open up discussions on the design space of HRI experiments and methodologies.

\section{ACKKNOWLEDGEMENT}

Many thanks go to our colleagues for participating in our trials; in particular, to Mike Blow for his acting and narration skills, and also his patience, in helping to produce the HRI video scenarios.

\section{REFERENCES}

[1] Fong, T., Nourbakhsh, I. and Dautenhahn, K. A survey of socially interactive robots. Robotics and Autonomous Systems, 42. 143-166.

[2] Te Boekhorst, R., Walters, M.L., Koay, K.L., Dautenhahn, K. and Nehaniv, C., A study of a single robot interacting with groups of children in a rotation game scenario. in Proc. IEEE CIRA 2005, (Finland, 2005). 35-40.
[3] Walters, M.L., Dautenhahn, K., Te Boekhorst, R., Koay, K.L., Kaouri, C., Woods, S., Nehaniv, C., Lee, D. and Werry, I., The influence of subjects' personality traits on personal spatial zones in a human-robot interaction experiment. in Proc. IEEE RO-MAN 2005, (Nashville, USA, 2005), 347-352.

[4] Walters, M.L., Dautenhahn, K., Te Boekhorst, R., Koay, K.L., Kaouri, C., Woods, S., Nehaniv, C., Lee, D. and Werry, I., The influence of subjects' personality traits on predicting comfortable human-robot approach distances. in Proc. COG SCI 2005; Towards Social Mechanisms of Android Science Workshop, (Stresa, Italy, 2005), 29-37.

[5] Severinson-Eklundh, K., Green, A. and Hüttenrauch, H. Social and collaborative aspects of interaction with a service robot. Robotics and Autonomous Systems, 42. 223-234.

[6] Kanda, T., Hirano, T., Eaton, D. and Ishiguro, H. Interactive robots as social partners and peer tutors for children: A field trial. Human Computer Interaction, 19 (1-2). 61-24.

[7] Robins, B., Dautenhahn, K. and Dubowski, J., Investigating Autistic children's attitudes towards strangers with the theatrical robot - A new experimental paradigm in human-robot interaction studies. in Proc. IEEE RO-MAN 2004, (Kurashiki, Japan, 2004), 557-562.

[8] Walters, M., Woods, S., Koay, K.L. and Dautenhahn, K., Practical and methodological challenges in designing and conducting interaction studies with human subjects. in Proc AISB'05, (University of Hertfordshire, Hatfield, UK, 2005), 110-119.

[9] Hinds, P., Roberts, T. and Jones, L. Whose job is it anyway? A study of human-robot interaction in a collaborative task. Human Computer Interaction, 19. 151-181.

[10] Dario, P., Guglieimelli, E. and Laschi, C. Humanoids and personal robots: Design and experiments. Journal of Robotic Systems, 18 (12). 673-690.

[11] Kanda, T. and Ishiguro, H. Reading human relationships from their interaction with an interactive humanoid robot. Lecture Notes in Computer Science, 3029. 402-412.

[12] Kanda, T., Ishiguro, H., Imai, M. and Ono, T., Body movement analysis of human-robot interaction. in IJCAI 2003, (2003), 177-182.

[13] Kidd, C.D. Sociable robots: The role of presence and task in humanrobot interaction Massachusetts Institute of Technology, Massachusetts, 2003.

[14] Shinozawa, K., Naya, F., Yamato, J. and Kogure, K. Differences in effect of robot and screen agent recommendations on human decision-making. IJHCS, 62 (2). 267-279.

[15] Paiva, A., Dias, J., Sobral, D., Aylett, R., Woods, S., Hall, L. and Zoll, C., Caring for Agents that care: Building empathic relations with synthetic agents. in Proc. AAMAS Autonomous Agents and Multi Agent Systems, (Columbia University, New York City, USA, 2004)

[16] .Murray, L. and Trevarthen, C. Emotional regulation of interaction between two-month-olds and their mothers. in Field, T. and Fox, N. eds. Social perception in infants, Norwood, NJ, USA, 1985, 101-125.

[17] Nadel, J., Carchon, I., Kervella, C. and Marcelli, D. Expectancies for social contingency in 2-month-olds. Developmental Science, 2 (2). 164-173.

[18] Dautenhahn, K., Woods, S., Kaouri, C., Walters, M., Koay, K.L. and Werry, I., What is a robot companion - Friend, assistant or butler? in Proc. IEEE IROS, (Edmonton, Canada, 2005), 1488-1493. 